International Journal of Pure and Applied Mathematics

Volume 88 No. 3 2013, 413-423

ISSN: $1311-8080$ (printed version); ISSN: 1314-3395 (on-line version)

url: http://www.ijpam.eu

doi: http://dx.doi.org/10.12732/ijpam.v88i3.9

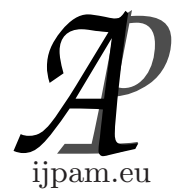

\title{
A HOCHSTADT-LIEBERMAN THEOREM FOR INTEGRO-DIFFERENTIAL OPERATOR
}

\author{
Murat Sat ${ }^{1}$, Emrah Yilmaz ${ }^{2}$ \\ ${ }^{1}$ Department of Mathematics \\ Erzincan University \\ Erzincan, 24100, TURKEY \\ ${ }^{2}$ Department of Mathematics \\ Firat University \\ Elazıg, 23119, TURKEY
}

\begin{abstract}
In this study, we solve a half inverse problem for integro-differential operator that consists of Sturm-Liouville differential part and integral part of Volterra type on a finite interval by using Hochstadt-Lieberman's method. We consider that $q$ is a squared integrable function on $[0, \pi]$ and the kernel of the integral perturbation is integrable on its domain. Especially, we show that the potential function $q$ is known as uniquely when the kernel of the integral perturbation is given.
\end{abstract}

AMS Subject Classification: 34B24, 34K29

Key Words: integro-differential equation, half inverse problem

\section{Introduction}

A classical regular Sturm-Liouville problem is related to a differential equation of the form

$$
-y^{\prime \prime}+q(x) y=\lambda y,
$$

with the following seperated boundary conditions

Received: July 16, 2013

(C) 2013 Academic Publications, Ltd.

${ }^{\S}$ Correspondence author 


$$
\left.\begin{array}{c}
y^{\prime}(0)-h y(0)=0 \\
y^{\prime}(\pi)+H y(\pi)=0
\end{array}\right\},
$$

where $q \in L_{2}(0, \pi)$ and $h, H$ are finite numbers. Inverse problems of spectral analysis consist in recovering operators from given some spectral datas. The first problem in this directon was solved by Ambarzumyan in 1929 [1]. This type of inverse problems were further solved by many authors with different methods [2], [3], [4], [5], [6], [7], [8]. Suppose that the spectrum $\left\{\lambda_{n}\right\}_{n \in \mathbb{N}}$ of Sturm-Liouville operator, the number $h$ or $H$ and the potential function $q$ on a half interval $\left(0, \frac{\pi}{2}\right)$ are known; one can recover the Sturm-Liouville operator based on this information. These problems are known as half-inverse problems in literature [9], [10], [11], [12], [13]. It was firstly solved by Hochstadt and Lieberman. They showed that if $q$ is known on $\left[\frac{\pi}{2}, \pi\right]$, then only one spectrum is sufficient to determine $q$ on $\left[0, \frac{\pi}{2}\right)$. Later, Hald [14] proved that if the potential function is known over half the interval and if one boundary condition is given, then the potential and the other boundary conditions are uniquely determined by using eigenvalues. This result generalizes a theorem which is given by Hochstadt and Lieberman. In [6] and [15], Gesztesy, Simons and Malamud gave some new uniqueness results in inverse spectral analysis with partial information on the potential function for scalar and matrix SturmLiouville equations, respectively. In 2001, Sakhnovich [12] studied existence of solution to the half-inverse problem; that is, he presented sufficient conditions on a function $\tilde{q}$ on $\left(0, \frac{1}{2}\right)$ and a sequence $\left\{\lambda_{n}^{2}\right\}$ of pairwise distinct real numbers tending to $+\infty$ in order that there existed a Sturm-Liouville operator on $(0,1)$ with given spectrum $\left\{\lambda_{n}^{2}\right\}$ and potential function $q$ coinciding with $\tilde{q}$ on $\left(0, \frac{1}{2}\right)$. In [10], authors gave the necessary and sufficient conditions for solvability of the half-inverse spectral problem for the class of Sturm-Liouville operators with singular potentials from the space $W_{2}^{-1}(0,1)$.

Let $\left\{\lambda_{n}\right\}_{n \geq 1}$ be the eigenvalues of a boundary value problem $L=L(q, M)$ of the form

$$
\begin{gathered}
L y(x)=-y^{\prime \prime}(x)+q(x) y(x)+\int_{0}^{x} M(x-t) y(t) d t=\lambda y(x), \lambda=\rho^{2}, x \in[0, \pi] \\
y(0)=y(\pi)=0,
\end{gathered}
$$

where $q$ and $M$ are real valued functions, $q \in L_{2}(0, \pi)$ and $M(x, t)$ is integrable on $[4],[16]$

$$
D_{0}=\left\{(x, t) \in R^{2}: 0 \leq t \leq x \leq \pi\right\}
$$


Now, we define

$$
\Delta(\lambda)=y(\pi, \lambda),
$$

which is called the charachteristic function of the boundary value problem (1.3), (1.4) where $\lambda$ is spectral parameter and $y\left(x, \lambda_{n}\right)$ are eigenfunctions of the problem (1.3), (1.4). This function has a countable set of zeros and the eigenvalues $\left\{\lambda_{n}\right\}_{n>1}$ of the boundary value problem (1.3), (1.4) coinicide with the zeros of the function $\Delta(\lambda)[4]$.

Lemma 1.1. (see [4], [16]) The functions $\varphi\left(x, \lambda_{n}\right)$ and $\psi\left(x, \lambda_{n}\right)$ are the eigenfunctions of the problem (1.3), (1.4) and there is a sequence $\left\{c_{n}\right\}_{n=0}^{\infty}$ such that

$$
\psi\left(x, \lambda_{n}\right)=c_{n} \varphi\left(x, \lambda_{n}\right), c_{n} \neq 0 .
$$

Lemma 1.2. (see [16]) The following relation holds

$$
\Delta(\lambda)=-\rho \sin (\rho \pi)+\omega \cos (\rho \pi)+r(\rho),
$$

where

$$
\omega=\frac{1}{2} \int_{0}^{\pi} q(x) d x
$$

and

$$
\begin{array}{r}
r(\rho)=\frac{1}{2} \int_{0}^{\pi} \cos [\rho(\pi-2 t)] q(t) d t+\int_{0}^{\pi} \cos (\rho t) d t \int_{t}^{\pi} M(\xi, t) \cos [\rho(\pi-\xi)] d \xi \\
+O\left(\frac{e^{|\tau| \pi}}{\rho}\right) .
\end{array}
$$

for $\tau=\operatorname{Im} \rho$.

Lemma 1. 3. (see [4], [16]) The eigenvalues $\left\{\lambda_{n}\right\}$ of the problem (1.3), (1.4) satisfy to the following relation

$$
\rho_{n}=\sqrt{\lambda_{n}}=n+\frac{\omega}{\pi n}+\frac{\kappa_{n}}{n}
$$

where

$$
\omega=\frac{1}{2} \int_{0}^{\pi} q(x) d x, \quad\left\{\kappa_{n}\right\} \in l_{2}, n=\overline{0, \infty} .
$$


uniformly in $x \in[0, \pi]$.

Lemma 1.4. (see [4]) The function $\Delta(\lambda)$ is uniquely determined by its zeros. Moreover,

$$
\Delta(\lambda)=\pi \prod_{n=1}^{\infty}\left(\frac{\lambda_{n}-\lambda}{n^{2}}\right) .
$$

The inverse problem consists in the reconstruction of the potential function $q$ from the spectra by the integro-differential expression for the problem (1.3), (1.4) where the function $M(x, t)$ is assumed to given. But, the classical methods are not always applicable in inverse problems for integro-differential operators. Whence, there are comperatively little references on inverse problem for integrodifferential operators. Nevertheless, some important results for this operator have been obtained by several authors [4], [16], [17], [18], [19], [20], [21], [22], [23].

In this study, the perturbation of the Sturm-Liouville operator by a Volterra type integral operator is considered. We give a half inverse problem for integrodifferential operator. And, by using Hochstadt-Lieberman method, we show that if the potential function $q$ and $M(x, t)$ are known on $\left[\frac{\pi}{2}, \pi\right]$ and $[0, \pi]$, respectively, then only one spectrum is also sufficient to determine the potential function $q$ as uniquely on $[0, \pi]$.

\section{Uniqueness Result}

In this section, we give a uniqueness theorem. Let consider another perturbation of Sturm-Liouville operator $\tilde{L}$ satisfying

$$
\widetilde{L} \tilde{y}=-\tilde{y}^{\prime \prime}+\widetilde{q}(x) \tilde{y}+\int_{0}^{x} M(x-t) \tilde{y}(t) d t,
$$

with the boundary conditions

$$
\widetilde{y}(0)=\tilde{y}(\pi)=0,
$$

where $\tilde{q}$ satisfies suitable regularity conditions on $[0, \pi]$.

Theorem 2.1. Let $\left\{\lambda_{n}\right\}_{n \geq 1}$ be the spectrum of the problem (1.3), (1.4) and $\left\{\widetilde{\lambda}_{n}\right\}_{n \geq 1}$ be the spectrum of the problem (2.1), (2.2). If $\lambda_{n}=\widetilde{\lambda}_{n}, n=1,2, \ldots$ and $\tilde{q}(x)=q(x)$ on $\left[\frac{\pi}{2}, \pi\right]$ then $\tilde{q}(x)=q(x)$ almost everywhere on $[0, \pi]$. 
Proof. Let consider following initial value problems

$$
\begin{gathered}
-y^{\prime \prime}+q(x) y+\int_{0}^{x} M(x-t) y(t) d t=\lambda y, \\
y(0)=0, y^{\prime}(0)=1
\end{gathered}
$$

and

$$
\begin{gathered}
-\widetilde{y}^{\prime \prime}+\tilde{q}(x) \tilde{y}+\int_{0}^{x} M(x-t) \tilde{y}(t) d t=\lambda \tilde{y} \\
\tilde{y}(0)=0, \tilde{y}^{\prime}(0)=1 .
\end{gathered}
$$

$\psi(x, \rho)$ and $\tilde{\psi}(x, \rho)$ are the solutions of $(2.3),(2.4)$ and (2.5), (2.6), respectively, for $\rho=\sqrt{\lambda} . \psi(x, \rho)$ and $\tilde{\psi}(x, \rho)$ have the following representations [4]

$$
\begin{aligned}
& \psi(x, \rho)=\frac{\sin (\rho x)}{\rho}+\int_{0}^{x} S(x, t) \frac{\sin (\rho t)}{\rho} d t, \\
& \tilde{\psi}(x, \rho)=\frac{\sin (\rho x)}{\rho}+\int_{0}^{x} \tilde{S}(x, t) \frac{\sin (\rho t)}{\rho} d t .
\end{aligned}
$$

respectively where $S(x, t)$ and $\tilde{S}(x, t)$ are continuous functions. Using $(2.7)$ and (2.8) for the solutions $\psi(x, \rho)$ and $\tilde{\psi}(x, \rho)$, we get

$$
\begin{aligned}
\psi \tilde{\psi}=\frac{\sin ^{2}(\rho x)}{\rho^{2}}+\int_{0}^{x}[S(x, t)+ & \widetilde{S}(x, t)] \frac{\sin (\rho x)}{\rho} \frac{\sin (\rho t)}{\rho} d t \\
& +\int_{0}^{x} S(x, t) \frac{\sin (\rho t)}{\rho} d t \int_{0}^{t} \tilde{S}(x, s) \frac{\sin (\rho s)}{\rho} d s .
\end{aligned}
$$

By using some trigonometric addition formulas and extending the range of $S(x, t)$ and $\widetilde{S}(x, t)$ with respect to $t$, we can obtain that

$$
\tilde{\psi}=\frac{1}{2 \rho^{2}}\left\{1-\cos (2 \rho x)+\int_{0}^{x} \hat{S}(x, \tau) \cos (2 \rho \tau) d \tau\right\}
$$


where

$$
\begin{aligned}
\hat{S}(x, \tau)=2[ & S(x, x-2 \tau)+\widetilde{S}(x, x-2 \tau) \\
& \left.\quad+\int_{-x+2 \tau}^{x} S(x, s) \tilde{S}(x, s-2 \tau) d s+\int_{-x}^{x-2 \tau} S(x, s) \tilde{S}(x, s+2 \tau) d s\right] .
\end{aligned}
$$

Now, we consider

$$
\Delta(\rho)=\psi(\pi, \rho) .
$$

Asymptotic results for $\psi(x, \rho)$ imply that this function is an entire function of order $\frac{1}{2}$ according to $\lambda$. Then $\Delta(\rho)$ is also an entire function of order $\frac{1}{2}$ according to $\lambda$. If we multiply (2.3) by $\psi$ and (2.5) by $\psi$ and substract after integration from 0 to $\pi$, we get

$$
\begin{aligned}
\left(\tilde{\psi} \psi^{\prime}-\tilde{\psi}^{\prime} \psi\right) & \left.\right|_{0} ^{\pi}+\int_{0}^{\pi} \int_{0}^{x} M(x-t)[\tilde{\psi}(t, \rho) \psi(x, \rho)- \\
& \psi(t, \rho) \tilde{\psi}(x, \rho)] d t d x \\
& +\int_{0}^{\pi}(\tilde{q}-q) \tilde{\psi} \psi d x=0 .
\end{aligned}
$$

and

$$
\begin{aligned}
\left(\tilde{\psi}(\pi, \lambda) \psi^{\prime}(\pi, \lambda)-\tilde{\psi}^{\prime}(\pi, \lambda) \psi(\pi, \lambda)\right) & \\
+\int_{0}^{\pi} \int_{0}^{x} M(x-t)[\tilde{\psi}(t, \rho) \psi(x, \rho)-\psi(t, \rho) \tilde{\psi}(x, \rho)] d t d x & \\
& =\int_{0}^{\frac{\pi}{2}}(q-\tilde{q}) \tilde{\psi} \psi d x .
\end{aligned}
$$

By virtue of (2.7) and its analogue for $\widetilde{L}$, we get

$$
\tilde{\psi}(t, \rho) \psi(x, \rho)-\psi(t, \rho) \tilde{\psi}(x, \rho)=O\left(\frac{1}{\rho^{2}}\right) .
$$

Then, we can rewrite (2.10) as 


$$
\begin{aligned}
\left(\tilde{\psi}(\pi, \lambda) \psi^{\prime}(\pi, \lambda)-\tilde{\psi}^{\prime}(\pi, \lambda) \psi(\pi, \lambda)\right)+\int_{0}^{\pi} \int_{0}^{x} M(x-t) O\left(\frac{1}{\rho^{2}}\right) d t d x & \\
& =\int_{0}^{\frac{\pi}{2}}(q-\tilde{q}) \tilde{\psi} \psi d x .
\end{aligned}
$$

Let define

$$
\int_{0}^{\frac{\pi}{2}}(q-\tilde{q}) \tilde{\psi}(x, \rho) \psi(x, \rho) d x=H(\rho)
$$

Using the properties of $\psi$ and $\tilde{\psi}$, we obtain that $H(\rho)$ is an entire function. For $\rho=\rho_{n}$, we see that the first and the second terms go to zero and hence $H\left(\rho_{n}\right)=0$ as $\rho \rightarrow \infty$. In addition, using (2.7) and (2.11), we see that

$$
|H(\rho)| \leq M
$$

for all complex $\rho$ and some constant $M$. Now, we consider quotient

$$
\Psi(\rho)=\frac{H(\rho)}{\Delta(\rho)} .
$$

It is evident that $\Psi(\rho)$ is an entire function and from (2.9) and (2.12)

$$
|\Psi(\rho)|=O\left(\frac{1}{\rho}\right),
$$

for large $|\rho|$. By the Liouville's theorem

$$
\Psi(\rho)=0 \text { or } H(\rho)=0 .
$$

for all $\rho$. Substituting (2.9) in (2.11), we yield

$$
\frac{1}{2 \rho^{2}} \int_{0}^{\frac{\pi}{2}}(q-\tilde{q})\left[1-\cos (2 \rho x)+\int_{0}^{x} \hat{S}(x, \tau) \cos (2 \rho \tau) d \tau \mid d x=0\right.
$$

for all $\rho$. We can change the order of integration where $0<\tau<x$ and $0<x<$ $\frac{\pi}{2}$. Hence last equation can be rewritten as 


$$
\int_{0}^{\frac{\pi}{2}}(q-\tilde{q}) d x+\int_{0}^{\frac{\pi}{2}} \cos (2 \rho \tau)\left\{[q(\tau)-\tilde{q}(\tau)]+\int_{\tau}^{\frac{\pi}{2}}[q(x)-\tilde{q}(x)] \hat{S}(x, \tau) d x\right\} d \tau
$$

Letting $\rho \rightarrow \infty$ for real $\rho$ and considering Riemann-Lebesque lemma, we obtain that

$$
\int_{0}^{\frac{\pi}{2}}[q(x)-\tilde{q}(x)] d x=0
$$

and

$$
\int_{0}^{\frac{\pi}{2}} \cos (2 \rho \tau)\left\{[q(\tau)-\tilde{q}(\tau)]+\int_{\tau}^{\frac{\pi}{2}}[q(x)-\tilde{q}(x)] \hat{S}(x, \tau) d x\right\} d \tau=0 .
$$

From the completeness of the functions $\cos (2 \rho \tau)$, the last integral must be vanish. Then, we get

$$
q(\tau)-\tilde{q}(\tau)+\int_{\tau}^{\frac{\pi}{2}}[q(x)-\tilde{q}(x)] \hat{S}(x, \tau) d x=0,
$$

for $0<\tau<\frac{\pi}{2}$. The last equation is a Volterra type integral equation and has only trivial solution. Then, we obtain that

$$
q(x)-\tilde{q}(x)=0
$$

almost everywhere on $[0, \pi]$. This completes the proof.

Remark. Let consider following initial value problems. If we assume that $\varphi(x, \lambda)$ and $\widetilde{\varphi}(x, \lambda)$ are the solutions of

$$
-y^{\prime \prime}+q(x) y+\int_{0}^{x} M(x-t) y(t) d t=\lambda y
$$




$$
y(0)=1, y^{\prime}(0)=0
$$

and

$$
\begin{gathered}
-\tilde{y}^{\prime \prime}+\tilde{q}(x) \tilde{y}+\int_{0}^{x} M(x-t) \tilde{y}(t) d t=\lambda \tilde{y} \\
\tilde{y}(0)=1, \tilde{y}^{\prime}(0)=0
\end{gathered}
$$

respectively, we can prove a uniqueness theorem for the problems (2.16), (2.17) and (2.18), (2.19) similarly.

\section{References}

[1] V. A. Ambartsumyan, Über eine frage der eigenwerttheorie, Zeitschrift für Physik, 53 (1929), 690-695.

[2] J. Pöschel and E. Trubowitz, Inverse spectral theory, Academic Press, Orlando, (1987).

[3] E. L. Isaacson, and E. Trubowitz, The inverse Sturm-Liouville problem. I., Communications on Pure and Applied Mathematics, 36 (1983), 767-783.

[4] G. Freiling and V. A. Yurko, Inverse Sturm-Liouville problems and their applications, NOVA Science Publishers, New York, (2001).

[5] I. M. Gelfand and B. M. Levitan, On the determination of a differential equation from its spectral function, American Mathematical Society Translations, 1 (1951), 253-304.

[6] F. Gesztesy, and B. Simon, Inverse spectral analysis with partial information on the potential. II: The case of discrete spectrum, Transactions of the American Mathematical Society, 3526 (2000), 2765-2787.

[7] H. Hochstadt, The inverse Sturm-Liouville problem, Communications on Pure and Applied Mathematics, 27 (1973), 715-729.

[8] W. Rundell and E. P. Sack, Reconstruction of a radially symetric potantial from two spectral sequences, Journal of Mathematical Analysis and Applications, 264 (2001), 354-381.

[9] H. Hochstadt and B. Lieberman, An inverse Sturm-Liouville problem with mixed given data, SIAM Journal on Applied Mathematics, 34 (1978), 676680 . 
[10] O. R. Hryniv and Y. V. Mykytyuk, Half inverse spectral problems for Sturm-Liouville operators with singular potentials, Inverse Problems, 20 5 (2004), 1423-1444.

[11] H. Koyunbakan and E. S. Panakhov, Half inverse problem for singular differential operator, Applicable Analysis, 84(3) (2005), 247-252.

[12] L. Sakhnovich, Half inverse problems on the finite interval, Inverse problems, 17 (2001), 527-532.

[13] C. F. Yang, and A. Zettl, Half inverse problems for quadratic pencils of Sturm-Liouville operators, Taiwanese Journal of Mathematics, 16(5), (2012), 1829-1846.

[14] O. Hald, Discontinuous inverse eigenvalue problems, Communications on Pure and Applied Mathematics, XXXVII (1984), 539-577.

[15] M. M. Malamud, Uniqueness questions in inverse problems for systems of differential equations on a finite interval, Transactions of the Moscow Mathematical Society, 60 (1999), 204-262.

[16] Y. V. Kuryshova and C. T. Shieh, An inverse nodal problem for integrodifferential operators, Journal of Inverse and Ill-posed Problems, 18 (2010), 357-369.

[17] Y. V. Kuryshova, Inverse spectral problem for integro-differential operators, Mathematical Notes, 816 (2007), 767-777.

[18] V. A. Yurko, Inverse problem for integro differential operators, Mathematical Notes,50 5 (1991), 1188-1197.

[19] V. A. Yurko, An inverse problem for integro-differential operators, Matematicheskie Zametki, 505 134-146 (Russian); English Translation in Mathematical Notes, 50 5-6 (1991), 1188-1197.

[20] S. A. Buterin, On an inverse spectral problem for a convolution integrodifferential operator, Results in Mathematics, 50 (2007), 173-181.

[21] C.T. Shieh, S.A. Buterin and M. Ignatiev, On Hochstadt-Lieberman theorem for Sturm-Liouville operators, Far East Journal of Applied Mathematics, 522 (2011), 131-146 
[22] Y. V. Kuryshova, An inverse spectral problem for differential operators with integral delay, Tamkang Journal of Mathematics, 423 (2011), 295303.

[23] E. S. Panakhov and M. Sat, Reconstruction of potential function for SturmLiouville operator with Coulomb potential, Boundary Value Problems, 49 (2013), 1-9. 
